\title{
Antimicrobial susceptibility of enterococci from Italian patients with infective endocarditis
}

\author{
Claudio Farina', Giuseppe Russello',Annibale Raglio², Francesca Vailati ${ }^{2}$,Veronica Ravasio ${ }^{3}$, Maria Bruna Pasticci, \\ Pierangelo Chinello ${ }^{5}$, Claudio Scarparo ${ }^{6}$, Gruppo SEI ${ }^{7}$ \\ I UOC Microbiologia e Virologia, AO Ospedale San Carlo Borromeo, Milano \\ 2 USC Microbiologia e Virologia, AO Ospedali Riuniti di Bergamo, Bergamo \\ 3 USC Malattie Infettive, AO Ospedali Riuniti di Bergamo, Bergamo \\ 4 Ospedale Santa Maria della Misericordia, Perugia; \\ $52^{\wedge}$ Divisione Malattie Infettive, INMI L. Spallanzani, Roma \\ 6 SOC Microbiologia, AOU S. Maria della Misericordia, Udine \\ 7 Gruppo SEl: elenco componenti in Appendice
}

Key words: Antimicrobial associations, Antimicrobial susceptibility, Enterococcus, Infective endocarditis

Profilo di antibioticosensibilità di enterococchi isolati da pazienti italiani con endocardite infettiva

\section{SUMMARY}

The epidemiological and clinical study "Studio Endocarditi Italiane" (SEI) evaluated 947 cases of infective endocarditis in 2004-2008. Enterococcal aethiology was described in $11 \%$ cases. The aim of our study was to evacuate the in vitro activity of twelve antibiotics alone and in association against 20 strains ( $15 \mathrm{E}$. faecalis, $5 \mathrm{E}$. faecium). Results show the in vitro activity of tigecyicline, daptomicine and linezolid. Synergism is appreciated for ceftriaxone-fosfomycin ( $F I C i n d e x 5_{0}=0.34$, FICindex ${ }_{90}=0.56$ ) against $E$. faecalis and for imipenem-fosfomycin against E. faecium. The majority of the tested strains show addition using ampicillin-ceftriaxone ( $F I C$ Cindex $50=0.85$, FICindex $90=1.0 \mathrm{I})$ and moxifloxacin-tigecycline ( $\left.\mathrm{FICindex} 5_{0}=0.77, \mathrm{FICindex} 90=1.96\right)$.

\section{INTRODUZIONE}

In corso di endocardite infettiva (EI) é fondamentale attuare un intervento terapeutico tempestivo e mirato. L'isolamento e l'identificazione dell'agente patogeno costituiscono il primo indispensabile passo per impostare correttamente la terapia, cui deve far seguito l'esecuzione di test di chemiosensibilità, la valutazione dell'attività battericida degli antibiotici e delle associazioni (15).

Lo studio multicentrico SEI (Studio Endocarditi Italiane) condotto in Italia (2004-2008) ha coinvolto 22 ospedali per delineare il quadro epidemiologico delle endocarditi infettive in Italia. Sono stati osservati 947 casi consecutivi di endocardite infettiva. Staphylococcus aureus è stato l'agente eziologico più frequente (24.5\%), seguito dagli streptococchi viridanti (19.2\%) e dagli stafilococchi coagulasi negativi (15\%). Enterococcus spp è stato isolato nell' $11 \%$ dei casi (5).

Negli ultimi anni si è assistito inoltre ad un incremento delle infezioni da Enterococci antibiotico-resistenti, sia in comunità che in ambito ospedaliero (3). La terapia delle endocarditi infettive enterococciche risulta, quindi, particolarmente difficile (14). In tale scenario la valutazione dell'efficacia di terapie combinate risulta di prioritaria importanza. Obiettivo dello studio è stata la valutazione in vitro dell'efficacia di 12 antibiotici singoli ed in associazione su Enterococcus spp. al fine di prospettare efficaci schemi di terapia combinata empirica, utilizzando tutti i ceppi isolati consecutivamente in quattro centri (Bergamo, Roma-Spallanzani, Perugia e Udine) che hanno partecipato allo studio microbiologico, in modo volontario, e aderenti allo studio clinico SEI di cui rappresentano una proiezione.

\section{MATERIALI E METODI}

Stipiti microbici. Sono stati saggiati 20 ceppi di Enterococcus spp (15 ceppi di E. faecalis e 5 di E. faecium), isolati da emocolture di pazienti affetti da endocardite infettiva, e pervenuti all'UOC di Microbiologia e Virologia dell’AO “Ospedale San Carlo Borromeo” di Milano nell'ambito dello studio clinico ed epidemiologico "SEI" coordinato dall'USC Malattie Infettive dell'AO “Ospedali Riuniti di Bergamo".

Gli stipiti microbici, sono stati conservati in criovials con sangue sterile di montone a $-80^{\circ} \mathrm{C}$ fino al momento del saggio e successivamente sottocoltivati in piastre di agar sangue di montone (AS Agar, bioMérieux s.a.) per consentire l'esecuzione dei test.

È stato utilizzato, come controllo, il ceppo E. faecalis ATCC 29212.

Agenti antimicrobici. Per ogni stipite microbico è stata determinata la MIC, utilizzando il metodo predefinito di gradiente E-Test (AB Biodisk, Solna, Svezia), nei confronti dei seguenti antibiotici (intervalli di concentrazione in $\mathrm{mg} / \mathrm{L}$ ): ampicillina (0.016-256), gentamicina e streptomicina ad alto dosaggio - HLAR - (0.064-1024), imipenem (0.002-32), ceftriaxone (0.016-256), vancomicina (0.016-256), linezolid (0.016-256), daptomicina (0.016-256), tigeciclina (0.016256), rifampicina (0.002-32) e moxifloxacina (0.002-32). Gli antibitiotici saggiati in associazione, mediante E- test sono riportati nella Tabella 1:

Tabella I. Antibiotici utilizzati in associazione

\begin{tabular}{rll}
\hline Antibiotici & Associazione \\
\hline $\mathbf{I}$ & Ampicillina (AMP) & HGM; HST; CRO; F \\
\hline $\mathbf{3}$ & Gentamicina HD (HGM) & LZ;VA; AMP \\
\hline $\mathbf{4}$ & Imipenem (IP) & LZ;VA; AMP \\
\hline $\mathbf{5}$ & Ceftriaxone (CRO) & LZ; F \\
\hline $\mathbf{6}$ & Vancomicina (VA) & AMP; F \\
\hline $\mathbf{7}$ & Linezolid (LZ) & RA; HST; HGM \\
\hline $\mathbf{8}$ & Daptomicina (DPC) & HGM; HST; IP; MX \\
\hline $\mathbf{9}$ & Tigeciclina (TGC) & MX \\
\hline I0 & Rifampicina (RA) & MX \\
\hline I I & Moxifloxacina (MX) & VA \\
\hline I 2 & Fosfomicina (F) & TGC; DPC; LZ \\
\hline
\end{tabular}

Determinazione della minima concentrazione inibente (MIC)

La MIC è stata determinata con il metodo predefinito di gradiente E-Test (AB Biodisk, Solna, Svezia) su piastre di Agar Mueller Hinton (bioMérieux s.a.) di $20 \mathrm{~cm}$ di diametro, inoculate con l'ausilio di un tampone sterile di rayon. Su ogni piastra sono state applicate 4 strisce di E-Test.

Le piastre sono state incubate overnight a $35^{\circ} \mathrm{C}$ in atmosfera

\section{Corresponding author: Claudio Farina}

UOC Microbiologia e Virologia, AO Ospedale San Carlo Borromeo

Milano, Via Pio II 3 - Tel.: 0240222456 - Fax 0240222829

E-mail: farina.claudio@sancarlo.mi.it 
aerobia. La lettura è stata effettuata in accordo con le linee guida fornite dal produttore.

Si è proceduto successivamente a calcolare, per ogni associazione microrganismo-antibiotico, il valore della MIC50 e della MIC9.

\section{Test delle associazione chemoantibiotiche}

I test di associazione sono stati determinati attraverso il metodo del rapporto fisso tra i gradienti (E-Test synergy screen method) che ha permesso di calcolare il Fractional Inhibitory Concentration Index ( $\Sigma$ FIC) per ciascun set di MIC. L'inoculo e la semina su piastre di Mueller Hinton (bioMérieux s.a) per ogni ceppo sono stati preparati come precedentemente descritto. Per valutare l'effetto delle associazioni, è stato successivamente calcolato l'indice FIC (Fractional Inhibitory Concentration), per ciascun antibiotico, in ciascuna combinazione.

Le associazioni sono definite sinergiche quando $\Sigma$ FIC $\leq 0.5$, antagoniste quando $\Sigma$ FIC $>4$, additive quando $\Sigma$ FIC è compreso tra 0.5 e 1 ed indifferente quando $\Sigma F I C$ è compreso tra $>1$ e $4(2,12-13)$. Si è proceduto successivamente a calcolare, per ogni associazione microrganismo-antibiotico, il valore del FICindex 50 e del FICindex 90.

\section{Analisi statistica}

I valori di MIC ottenuti sono stati trasformati in valori logaritmici al fine di approssimare una normale distribuzione prima dell'analisi statistica. Le variabili continue sono state comparate con il t-test Student's. È stata anche calcolata la media e la deviazione standard sia delle MIC, sia in singolo che in associazione. I risultati significativi sono stati valutati attraverso l'analisi della varianza Bonferroni t-test. Un valore di $p<0.05$ è stato considerato significativo.

\section{RISULTATI}

La lettura dei test di chemioantibiticosensibilità effettuati tramite E-test, ha evidenziato ellissi di inibizione uniformi e ben delineate. I valori di MIC per i 20 ceppi di Enterococcus spp. saggiati sono compendiati nella tabella 2 . Le tabelle 3 e 4 compendiano i risultati dei saggi di associazione effettuati nei confronti dei soli stipiti di $E$. faecalis, in quanto l'elevata farmaco-resistenza di E. faecium non ha consentito di evidenziare valori FIC index significativi.

Tutti i ceppi di Enterococcus spp. sono risultati sensibili a tigeciclina (range: $0.032-0.25 \mu \mathrm{g} / \mathrm{ml}, \mathrm{MIC}_{50}=0.125$ e MIC $_{90}=0.25$ ), daptomicina, (range: $0.047-1.5 \mu \mathrm{g} / \mathrm{ml}$,
MIC $_{50}=0.25$ e MIC $_{90}=1.5$ ) e linezolid (range: $0.125-0.75$ $\mu \mathrm{g} / \mathrm{ml}, \mathrm{MIC}_{50}=0.5$ e MIC $\left.{ }_{90}=0.75\right)$.

L'85\% (17/20) degli isolati è risultato sensibile a vancomicina (range: $0.5->256 \mu \mathrm{g} / \mathrm{ml}, \mathrm{MIC}_{50}=1,5$ e $\mathrm{MIC}_{90}=>256$ ), l'80\% (16/20) ad ampicillina (range: 0.064 - >256 $\mu \mathrm{g} / \mathrm{ml}$, $\mathrm{MIC}_{50}=0,5$ e $\mathrm{MIC}_{90}=256$ ), gentamicina (range: $1.5->1024$ $\mu \mathrm{g} / \mathrm{ml}, \mathrm{MIC}_{50}=16$ e $\mathrm{MIC}_{90}=>1024$ ) e a rifampicina (range: $0.012-32 \mu \mathrm{g} / \mathrm{ml}$, MIC $_{50}=0.5$ e MIC $\left.{ }_{90}=4\right)$, il 75\% (15/20) a imipenem (range: $0.064->32 \mu \mathrm{g} / \mathrm{ml}, \mathrm{MIC}_{50}=1.5$ e

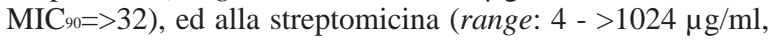
MIC $_{50}=128$ e MIC $\left._{90}=>1024\right)$, il 70\% (14/20) alla moxifloxacina (range: $0.064->32 \mu \mathrm{g} / \mathrm{ml}, \mathrm{MIC}_{50}=0.25$ e $\mathrm{MIC}_{90}=32$ ), il $65 \%(13 / 20)$ a fosfomicina (range: 16 - >1024 $\mu \mathrm{g} / \mathrm{ml}$, $\mathrm{MIC}_{50}=32$ e $\left.\mathrm{MIC}_{90}=192\right)$. Il $100 \%$ dei ceppi è risultato resistente al ceftriaxone (range da $32 \mathrm{a}>256 \mu \mathrm{g} / \mathrm{ml}$, MIC ${ }_{50}=>256$ e $\left.\mathrm{MIC}_{90}=>256\right)$.

Per quanto attiene all'associazione ampicillina-ceftriaxone è stata riscontrata una riduzione significativa della media geometrica della MIC del ceftriaxone (da 68.9 a $0.25 \mu \mathrm{g} / \mathrm{ml}$; $p<0.01$ ) ma non dell'ampicillina (da 0.31 a $0.26 \mu \mathrm{g} / \mathrm{ml} ; p=0.4$ ). Nel $13.4 \%$ dei casi l'associazione dei due antibiotici è risultata sinergica, nel $73.3 \%$ additiva e nell'13.3\% indifferente. Non è stato osservato nessun effetto antagonista. La maggioranza dei ceppi saggiati presentava valori FIC index di 1.01 .

Per quanto concerne l'associazione ceftriaxone-fosfomicina. è stata riscontrata una riduzione significativa della media geometrica sia della MIC del ceftriaxone (da 68.9 a 12.7 $\mu \mathrm{g} / \mathrm{ml} ; p=<0.01$ ) che della fosfomicina (da 0.31 a $0.26 \mu \mathrm{g} / \mathrm{ml}$; $p<0.01$ ). Nel $60 \%$ dei casi l'associazione dei due antibiotici è risultata sinergica, nel $26.7 \%$ dei casi additivi e nel $13.3 \%$ dei casi indifferente. La maggioranza dei ceppi saggiati presentava valori FIC index di 0.56 .

Per quanto concerne l'associazione ampicillina-streptomicina è stata rilevata una riduzione significativa della media della MIC della streptomicina (da 146.5 a $0.35 \mu \mathrm{g} / \mathrm{ml} ; p<0.01$ ) ed un incremento significativo della media geometrica della MIC dell'ampicillina (da 0.31 a $1.4 \mu \mathrm{g} / \mathrm{ml} ; p<0.01$ ). Nel $6.7 \%$ dei casi l'associazione dei due antibiotici è risultata sinergica, nel 46.7\% dei casi additiva ed indifferente. La maggioranza dei ceppi saggiati presentava valori FIC index di 1.51 .

L'associazione linezolid-streptomicina ha evidenziato una riduzione significativa della media geometrica della MIC della streptomicina (da 146.5 a $0.56 \mu \mathrm{g} / \mathrm{ml} ; p<0.01$ ) ed un incremento significativo della media della MIC del linezolid (da 0.51 a $2.32 \mu \mathrm{g} / \mathrm{ml} ; p<0.01$ ). Nel $53.3 \%$ dei casi l'associazione dei due antibiotici è risultata additiva e nel $46.7 \%$ dei casi indifferente. La maggioranza dei ceppi saggiati presenta-

Tabella 2. Valori di MIC $(\mu \mathrm{g} / \mathrm{ml})$ dei 20 ceppi di Enterococcus spp. saggiati

\begin{tabular}{|c|c|c|c|c|c|c|c|c|c|c|c|c|c|}
\hline $\mathbf{N}^{\circ}$ & Ceppi & AMP & HGM & HST & IMP & CRO & VA & $\mathbf{L Z}$ & DAP & TIG & RA & MOX & $\mathbf{F}$ \\
\hline$I$ & E. faecalis & 0.38 & 12 & 128 & 0.75 & 48 & 3 & 0.75 & 0.125 & 0.19 & 1.5 & 0.19 & 16 \\
\hline 2 & E. faecalis & 0.5 & 24 & 256 & 1.5 & 32 & 1 & 0.75 & 0.25 & 0.25 & 2 & 0.19 & 16 \\
\hline 3 & E. faecalis & 0.38 & 8 & 48 & 1 & $>256$ & 2 & 0.5 & 0.25 & 0.064 & 0.5 & 0.25 & 16 \\
\hline 4 & E. faecalis & 0.5 & 16 & 256 & 1.50 & 48 & 3 & 0.75 & 0.25 & 0.25 & 0.75 & 0.38 & 16 \\
\hline 5 & E. faecalis & 0.5 & 16 & 192 & 1.5 & 64 & $\mathrm{I}$ & 0.75 & 0.125 & 0.19 & 1.5 & 0.19 & 24 \\
\hline 6 & E. faecalis & 0.19 & 8 & 96 & 0.5 & $>256$ & 2 & 0.75 & 0.38 & 0.125 & 0.38 & 0.25 & 24 \\
\hline 7 & E. faecalis & 0.38 & 16 & $>1024$ & 1.5 & $>256$ & 1 & 0.75 & 0.19 & 0.25 & 0.5 & 0.25 & 24 \\
\hline 8 & E. faecalis & 0.38 & 12 & 128 & I & $>256$ & 1.5 & 0.75 & 0.19 & 0.19 & 4 & 0.25 & 24 \\
\hline 9 & E. faecalis & 0.064 & 1.5 & 4 & 0.064 & 48 & 0.5 & 0.125 & 0.19 & 0.064 & 0.012 & 0.064 & 32 \\
\hline 10 & E. faecalis & 0.125 & 16 & 192 & $\mathrm{I}$ & $>256$ & 2 & 0.75 & 0.25 & 0.25 & 1 & 0.19 & 32 \\
\hline 11 & E. faecalis & 0.5 & 12 & 96 & 1.50 & $>256$ & 1.5 & 0.75 & 0.38 & 0.125 & 3 & 0.25 & 32 \\
\hline 12 & E. faecalis & 0.5 & $>1024$ & $>1024$ & 1 & $>256$ & 1.5 & 0.5 & 0.047 & 0.25 & 0.5 & $>32$ & 96 \\
\hline 13 & E. faecalis & 0.25 & 4 & 128 & 0.50 & 64 & $\mathrm{I}$ & 0.125 & 0.094 & 0.094 & 0.19 & 0.38 & 192 \\
\hline 14 & E. faecalis & 0.5 & 24 & 256 & 2 & $>256$ & 2 & 0.5 & 0.19 & 0.19 & 0.38 & 0.19 & $>1024$ \\
\hline 15 & E. faecalis & 0.25 & $>1024$ & 128 & 2 & $>256$ & 1.5 & 0.25 & 0.19 & 0.094 & 0.125 & 12 & $>1024$ \\
\hline 16 & E. faecium & 0.38 & 3 & 32 & 16 & $>256$ & 0.5 & 0.19 & 1.5 & 0.032 & 0.25 & 4 & 24 \\
\hline 17 & E. faecium & $>256$ & $>1024$ & $>1024$ & $>32$ & $>256$ & $>256$ & 0.38 & 1 & 0.125 & 4 & $>32$ & 48 \\
\hline 18 & E. faecium & $>256$ & $>1024$ & $>1024$ & $>32$ & $>256$ & $>256$ & 0.38 & 1 & 0.125 & 12 & $>32$ & 64 \\
\hline 19 & E. faecium & $>256$ & 6 & 48 & $>32$ & $>256$ & 2 & 0.75 & 1.5 & 0.125 & 32 & $>32$ & 96 \\
\hline 20 & E. faecium & $>256$ & 256 & $>1024$ & $>32$ & $>256$ & $>256$ & 0.5 & 1.5 & 0.094 & 0.19 & 32 & 128 \\
\hline & $M / C_{50}$ & 0.5 & 16 & 128 & 1.5 & $>256$ & 1.5 & 0.5 & 0.25 & 0.125 & 0.5 & 0.25 & 32 \\
\hline & $M I C_{50}$ & $>256$ & $>1024$ & $>1024$ & $>32$ & $>256$ & $>256$ & 0.75 & 1.5 & 0.25 & 4 & $>32$ & 192 \\
\hline
\end{tabular}


va valori FIC index di 1.51

Per quanto concerne l'associazione moxifloxacina-tigeciclina è stata rilevata una riduzione significativa della media geometrica della MIC della moxifloxacina (da 0.39 a $0.09 \mu \mathrm{g} / \mathrm{ml}$; $p<0.01$ ) e della tigeciclina (da 0.15 a $0.017 \mu \mathrm{g} / \mathrm{ml} ; p<0.01$ ). Nel 66.6\% dei casi l'associazione dei due antibiotici è risultata additiva e nel 33.3\% dei casi indifferente. La maggioranza dei ceppi saggiati presentava valori FIC index di 1.96.

Dati contrastanti sono stati ottenuti per quanto riguarda l'associazione imipenem-fosfomicina considerando separatamente i ceppi di E. faecalis e quelli di E. faecium. E stato infatti riscontrato un effetto antagonista sostenuto dall'associazione dei due antibiotici nella maggior parte dei ceppi $E$. faecalis (60\% dei casi) ed un effetto sinergico nella totalità dei ceppi di $E$. faecium saggiati.

Non sono state osservate riduzioni significative delle medie geometriche delle MIC dei test in associazione rispetto a quelle ottenute con le stesse molecole valutate in singolo per le rimanenti associazioni effettuate.

\section{CONCLUSIONI}

Nel 1999 Gutschik dimostrò che l'associazione di aminoglicosidi e betalattamine determina un effetto battericida sinergico su Enterococcus spp. (9). Da allora la terapia di elezione delle endocarditi enterococciche si è basata sull'associazione di penicillina con streptomicina o gentamicina (1). Tuttavia, la crescente comparsa di ceppi resistenti a penicillina o ad ampicillina, oppure altamente resistenti agli aminoglicosidi e, più tardi, ai glicopeptidi, ha ulteriormente complicato l'approccio terapeutico (9). Non più eccezionale è il riscontro di Enterococcus spp. resistente ai glicopeptidi o con un'alta resistenza alla gentamicina (MIC gentamicina $>500$ $\mu \mathrm{g} / \mathrm{ml}$ ) ed alla streptomicina (MIC streptomicina $>1500$ $\mu \mathrm{g} / \mathrm{ml})$. In questi casi, le opzioni terapeutiche sono oggi rappresentate da linezolid e quinopristin/dalfopristin oppure da associazioni di antibiotici scelte sulla base dei test di sensibilità in vitro (6).

In tale scenario la valutazione dell'efficacia della terapia antibiotica di associazione risulta di prioritaria importanza. In letteratura è riportata l'efficacia in vivo ed in vitro dell'associazione di ampicillina e ceftriaxone nel caso di ceppi di Enterococcus faecalis con elevata resistenza agli aminoglicosidi. Gavalda et al. (8) mostrano, ad esempio, come l'associazione ampicillina-ceftriaxone $(4 \mu \mathrm{g} / \mathrm{ml})$ determini una riduzione della MIC dell'ampicillina di 1-4 logaritmi e come le curve di time/killing dell'ampicillina (1-2 $\mu \mathrm{g} / \mathrm{ml})$ in associazione con concentrazioni crescenti (da 5 a $60 \mu \mathrm{g} / \mathrm{ml}$ ) di ceftriaxone pre- sentino una riduzione di $\geq 2 \log _{10} \mathrm{CFU} / \mathrm{ml}$ rispetto a quelle dell'ampicillina testata singolarmente. Gli studi effettuati utilizzando modelli animali mostrano inoltre come la concentrazione batterica residua nel contesto di vegetazioni sperimentalmente indotte sulla valvola aortica risulti significativamente più bassa negli animali trattati con ampicillina-ceftriaxone che in quelli trattati con ampicillina in monoterapia (8).

I dati della nostra valutazione evidenziano come nel 13\% dei casi l'associazione di ampicillina e ceftriaxone sia sinergica. D'altra parte, qualora non venga rilevato un effetto sinergico, questa associazione determina un effetto comunque vantaggioso perché additivo (73.3\% dei casi). La terapia combinata determina infatti una riduzione significativa della media geometrica della MIC del ceftriaxone (da 68.9 a $0.25 \mu \mathrm{g} / \mathrm{ml}$; $p<0.01$ ) ma non dell'ampicillina (da 0.31 a $0.26 \mu \mathrm{g} / \mathrm{ml}$; $\mathrm{P}=0.4$ ), risultando comunque più efficace rispetto alla monoterapia.

Heather et al. hanno mostrato, in modelli animali, come l'associazione ampicillina-gentamicina non determini - nei confronti di E. faecalis - una riduzione significativa della carica batterica rispetto all'ampicillina utilizzata singolarmente (10). Al contrario, Fass et al. concludono che l'associazione ampicillina-gentamicina determina una riduzione significativa della carica batterica rispetto all'ampicillina utilizzata singolarmente (7). Nonostante la letteratura scientifica mostri dati contrastanti, l'associazione di ampicillina con un aminoglicoside è raccomandata dall'American Heart Association per il trattamento delle endocarditi enterococciche (2).

I nostri dati mostrano come, in vitro, l'associazione ampicillina-gentamicina risulti indifferente sulla maggioranza dei ceppi, sia considerando il genere Enterococcus che la specie E. faecalis. La terapia combinata non determina un vantaggio rispetto alla monoterapia. Per quanto concerne l'associazione ampicillina-streptomicina, solo nel $6.7 \%$ dei casi l'associazione risulta sinergica, determinando tuttavia un effetto additivo nel $46.7 \%$ circa dei casi. La terapia combinata determina inoltre una riduzione significativa della media geometrica della MIC della streptomicina (da 146.5 a $0.35 \mu \mathrm{g} / \mathrm{ml} ; p<0.01$ ) ed un incremento della media della MIC dell'ampicillina (da $0.31 \mathrm{a} 1.4 \mu \mathrm{g} / \mathrm{ml} ; p<0.01)$. Anche se l'associazione vancomicina-aminoglicoside è raccomandata dall'American Heart Association nel caso di endocarditi infettive da ceppi resistenti alla penicillina (2), i nostri risultati mostrano come le associazioni di vancomicina con gentamicina o streptromicina ad alto dosaggio risultino indifferenti.

Scarsi, al contrario, sono i dati riportati dalla letteratura internazionale in merito all'associazione imipenem-linezolid e riguardano, nella maggior parte dei casi, Staphylococcus

Tabella 3. Percentuale di additività, indifferenza, sinergia ed antagonismo delle diverse associazioni antibiotiche verso $i$ ceppi di E. faecalis

\begin{tabular}{lcccc}
\hline ASSOCIAZIONI & ADDITIVITA & INDIFFERENZA & SINERGIA & I \\
\hline AMP+CRO & $73.3 \%$ & $13.3 \%$ & 0 & 0 \\
\hline RA+VA & $20 \%$ & $80 \%$ & 0 & 0 \\
\hline MOX+TGC & $66.7 \%$ & $33.3 \%$ & 0 & 0 \\
\hline MOX+DPC & $13.3 \%$ & $66.7 \%$ & 0 & 0 \\
\hline MOX+LZ & $46.7 \%$ & $46.7 \%$ & 0 & 0 \\
\hline IP+LZ & $20 \%$ & $73.3 \%$ & $6.6 \%$ & $6 \%$ \\
\hline IP+F & 0 & $40 \%$ & $60 \%$ & 0 \\
\hline $\mathbf{C R O + F}$ & $26.7 \%$ & $13.3 \%$ & 0 \\
\hline AMP+F & $13.3 \%$ & $86.7 \%$ & 0 \\
\hline
\end{tabular}

Tabella 4. Valori di FICindex 50 e di FICindex 90 dei 15 ceppi di E. faecalis saggiati

\begin{tabular}{|c|c|c|c|c|c|c|c|c|c|c|c|c|c|c|}
\hline AMP & AMP & AMP & LZ & VA & RA & LZ & VA & MOX & MOX & MOX & IMP & IMP & CRO & AMP \\
\hline+ & + & + & + & + & + & + & + & + & + & + & + & + & + & + \\
\hline CRO & HGM & HST & HST & HST & VA & HGM & HGM & TIG & DAP & $\mathbf{L Z}$ & $\mathbf{L Z}$ & $\mathbf{F}$ & $\mathbf{F}$ & $\mathbf{F}$ \\
\hline \multicolumn{15}{|c|}{ FICindex50 } \\
\hline 0.85 & 1.12 & 1.00 & 1.02 & $\mathrm{I} .4 \mathrm{I}$ & 1.50 & $\mathrm{I} .12$ & 1.50 & 0.77 & 1.18 & 1.03 & 1.07 & 4.08 & 0.34 & 1.41 \\
\hline Add* & Ind* & Add & Add & Ind & Ind & Ind & Ind & Add & Ind & Add & Ind & Ant* & Sin* & Ind \\
\hline \multicolumn{15}{|c|}{ FICindex90 } \\
\hline 1.01 & 1.58 & 1.51 & 1.51 & 2.06 & 2.16 & 1.69 & 2.00 & 1.96 & 6.16 & 2.27 & 1.60 & 7.93 & 0.56 & 1.62 \\
\hline Add & Ind & Ind & Ind & Ind & Ind & Ind & Ind & Ind & Ant & Ind & Ind & Ant & Add & Ind \\
\hline
\end{tabular}

*Add: associazione additiva; Sin: associazione sinergica; Ind: associazione indifferente;Ant: associazione antagonista 
aureus: essi riportano un effetto indifferente o, addirittura, di moderato antagonismo (11). I nostri dati non si discostano da questi, mostrando nella maggior parte dei casi un effetto indifferente ed in minima parte un effetto antagonista. In mancanza di dati riportati dalla letteratura scientifica, innovative parrebbero le associazioni moxifloxacina-linezolid e linezolid-streptomicina. In realtà l'associazione moxifloxacina-linezolid è risultata prevalentemente indifferente, mentre linezolid-streptomicina pare, nel 53.3\% dei casi, additiva. È stata anche osservata una riduzione significativa della media della MIC della streptomicina (da 146.5 a $0.56 \mu \mathrm{g} / \mathrm{ml}$; $p<0.01)$ ed un incremento della media della MIC del linezolid (da 0.51 a $2.32 \mu \mathrm{g} / \mathrm{ml} ; p<0.01$ ). Questi risultati dimostrano la necessità di approfondire ulteriormente, con ulteriori studi in vitro, l'efficacia dell'associazione linezolid-streptomicina, in particolar modo nei confronti di E. faecalis. L'associazione linezolid-gentamicina per alti livelli di resistenza agli aminoglicosidi risulta poi indifferente: linezolid, tuttavia, ha mostrato un'eccellente attività in vitro nei confronti di Enterococcus spp. che ne lascia prefigurare, in virtù delle sue caratteristiche di elevata diffusibilità tissutale, l'utilizzo terapeutico (2).

La valutazione dell'efficacia in vitro delle associazioni rifampicina-vancomicina, moxiciclina- daptomicina, moxiciclinatigeciclina, imipenem-fosfomicina, ceftriaxone-fosfomicina ed ampicillina-fosfomicina risulta particolarmente innovativa, in quanto la letteratura internazionale non riporta dati in merito. I nostri risultati mostrano come le associazioni rifampicina-vancomicina ed ampicillina-fosfomicina risultino indifferenti nella quasi totalità dei casi, sia considerando il genere Enterococcus spp. nel suo insieme sia la sola specie $E$. faecalis. Analogamente, nella maggior parte dei casi è indifferente o debolmente antagonista l'associazione moxifloxacina-daptomicina.

Da sottolineare sono i risultati ottenuti con l'associazione moxifloxacina-tigeciclina. Questa associazione è prevalentemente additiva (66.7\% dei casi), determinando anche una significativa riduzione della media geometrica della MIC della moxifloxacina (da 0.39 a $0.09 \mu \mathrm{g} / \mathrm{ml} ; p<0.01$ ) e della tigeciclina (da 0.15 a $0.017 \mu \mathrm{g} / \mathrm{ml} ; p<0.01$ ) proponendosi come schema terapeutico potenzialmente efficace.

Studi precedenti avevano evidenziato l'effetto sinergico dell'associazione imipenem-fosfomicina nel 50\% dei ceppi di $E$. faecalis e nel $60 \%$ di E. faecium (4). I dati della nostra valutazione hanno evidenziato un effetto antagonista nella maggior parte dei ceppi $E$. faecalis (60\% dei casi) ma un effetto sinergico nella totalità dei ceppi di E. faecium saggiati.

Da sottolineare sono infine i risultati ottenuti con l'associazione fosfomicina-ceftriaxone. I nostri dati mostrano come nel $60 \%$ dei casi l'associazione dei due farmaci sia sinergica e qualora non venga rilevato un effetto sinergico questa associazione determina un effetto additivo in un altro $26.7 \%$ dei ceppi. La terapia di associazione determina inoltre una riduzione significativa della media geometrica della MIC del ceftriaxone (da 68.9 a $12.7 \mu \mathrm{g} / \mathrm{ml} ; p<0.01$ ) e della fosfomicina (da 0.31 a $0.26 \mu \mathrm{g} / \mathrm{ml} ; p<0.01$ ). La terapia combinata in questi casi determina un significativo vantaggio rispetto alla terapia in singolo.

Considerata la scarsità di dati, si sottolinea la necessità di procedere ad ulteriori approfondimenti relativamente all'associazione ceftriaxone-fosfomicina (in particolare nei confronti di $E$. faecalis) e ad imipenem-fosfomicina (in particolare per $E$. faecium). Particolarmente significativo appare il ruolo - in associazione - di fosfomicina che si mostra antibiotico di particolare interesse tale da lasciarne ipotizzare l'impiego in terapia nelle EI da E. faecium: si segnala tuttavia che questa mole- cola non è disponibile in Italia e che il suo impiego nella terapia dell'EI sarebbe off label. La tigeciclina, normalmente utilizzata nelle infezioni della cute, tessuti molli ed endoaddominali, ha inoltre mostrato un'eccellente attività in vitro sia singolarmente che in associazione con moxifloxacina e, per la sua ottima diffusibilità tissutale, potrebbe anche essere utilizzabile nella terapia di EI enterococciche.

\section{Appendice: Il Gruppo SEI}

Suter F, Rizzi M, Ravasio V, AO Ospedali Riuniti di Bergamo; Biglino A, Ospedale Civile di Asti; Carosi G, Università degli Studi di Brescia; Persiani M, Ospedale di Cuneo; Utili R, AO Monaldi, Napoli; Tripodi MF, II Univ., Policlinico Universitario di Napoli; Minoli L, IRCCS Policlinico San Matteo, Pavia; Petrosillo N, INMI “L. Spallanzani”, Roma; Venditti M, Policlinico Umberto I, Roma; Vaglia A, Ospedale S. Maria di Ca' Foncello, Treviso; Crapis M, Policlinico Universitario, Udine; Grossi $P$, Università degli Studi dell'Insubria, Varese; Concia E, Università degli Studi di Verona.

\section{BIBLIOGRAFIA}

1. Baddour LM, Wilson WR, Bayer AS, et al. Infective Endocarditis: Diagnosis, Antimicrobial Therapy, and Management of Complications. A Statement for Healthcare Professionals from the Committee on Rheumatic Fever, Endocarditis, and Kawasaki Disease, Council on Cardiovascular Disease in the Young, and the Councils on Clinical Cardiology, Stroke, and Cardiovascular Surgery and Anesthesia, American Heart Association Executive. Summary: Endorsed by the Infectious Diseases Society of America. Circulation, 2005; 111: 3167-84.

2. Bolmström A, Arvidson S, Ericsson M, Karlsson A. New in vitro models for studying antibiotic combinations using predefined antibiotic gradients. ICAAC, 1989 (Poster).

3. Concia E, Farina C, Marone P. Il test di batteriocidia nel trattamento delle infezioni gravi. Boll. Bibl. OORR BG, 1983; 2: 41-2.

4. Debbia E, Varaldo PE, Schito GC. In vitro activity of imipenem against enterococci and staphylococci and evidence for high rates of synergism with teicoplanin, fosfomycin, and rifampin. Antimicrob Agents Chemother. 1986; 30: 813-5.

5. Eliopoulus GM. Enterococcal endocarditis. In: Kaye D, ed. Infective endocarditis. New York, NY: Raven Press, 1992; 209-29.

6. Elliott TSJ, Foweraker J, Gould FK, Perry JD, Sandoe JAT. Guidelines for the antibiotic treatment of endocarditis in adults: report of the Working Party of the British Society for Antimicrobial Chemotherapy. J Antimicrob Chemother 2004; 54: 971-81.

7. Fass RJ, Wright CA. Comparative efficacies of mezlocillin and ampicillin alone or in combination whit gentamicin in the treatment of Streptococcus faecalis endocarditis in rabbits. Antimic Agent Chemother, 1984; 25: 40810

8. Gavalda J, Torres C, Tenorio C, et al. Efficacy of ampicillina plus ceftriaxone in treatment of experimental endocarditis due to Enterococcus faecalis strains highly resistant to aminoglycosides. Antimic Agents Chemother 1999; 43: 639-48.

9. Gutschik E. New developments in the treatment of infective endocarditis and infective cardiovasculitis. Int Antimicrob Agents 1999; 13: 79-83.

10. Heather H, Houlihan C, Daniela P, Rybak MJ. Pharmacodynamics of vancomycin and ampicillin alone and in combination with gentamicin once daily or thrice daily against Enterococcus faecalis in an in vitro infection model. J Antimicrob Chemother 2000; 46, 79-86.

11. Jacqueline C, Navas D, Batard E, et al. In vitro and in vivo synergistic activities of linezolid combined with subinhibitory concentrations of imipenem against methicillin-resistant Staphylococcus aureus. Antimicrob Agents Chemother, 2005; 49: 45-51.

12. Lewis R, Diekema D, Messer S, Pfaller MA, Klepser ME. Comparison of Etest, chequerboard dilution and time kill studies for the detection of synergy or antagonism between antifungal agents tested against Candida species. J Antimicrob Chemother, 2002; 49: 345-51.

13. Manno G, Ugonotti E, Belli M, Fenu ML, Romano L, Cruciani M. Use of the $\mathrm{E}$ test to assess synergy of antibiotic combinations against isolates of Burkholderia cepacia- complex from patients with Cystic Fibrosis. EJCMID, 2003; 22: 28-34.

14. National Nosocomial Infections Surveillance (NNIS) report, data summary from October 1986-April 1996, issued May 1996. A report from the National Nosocomial Infections Surveillance (NNIS) System. Am J Infect Control, 1996; 24: 380-8.

15. Ravasio V, Rizzi M, Stellini R, et al. Current trends in infective endocarditis in Italy: report on 852 cases from the multicenter, prospective SEI study. Abstract. 